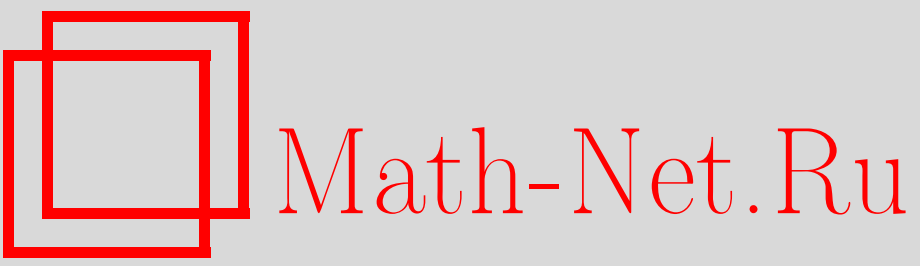

С. В. Савченко, О максимальных подорграфах с наибольшим числом перрона, УМH, 2001, том 56, выпуск 6, 165-166

DOI: https://doi.org/10.4213/rm468

Использование Общероссийского математического портала Math-Net.Ru подразумевает, что вы прочитали и согласны с пользовательским соглашением

http://www.mathnet.ru/rus/agreement

Параметры загрузки:

IP : 34.239 .49 .27

26 апреля 2023 г., 08:32:52 


\title{
О МАКСИМАЛЬНЫХ ПОДОРГРАФАХ С НАИБОЛЬШИМ ЧИСЛОМ ПЕРРОНА
}

\author{
C. В. САВЧЕНКО
}

Любой максимальныи подорграф ориентированного графа (орграфа) $G$ (с множеством вершин $V(G)$ и множеством дуг $E(G))$ имеет вид $G-w$ и получается из $G$ удалением некоторой вершины $w$ и всех инцидентных ей дуг (в нашей работе мы будем придерживаться определений главы 3 книги [1]). Обозначим через $\lambda(G-w)$ число Перрона орграфа $G-w$, которое, по определению, совпадает со спектральным радиусом его матрицы смежности. В дальнейшем мы будем рассматривать максимальные подорграфы с наибольшим числом Перрона (максимальные подорграфы с наименьшим числом Перрона были исследованы в [2]). Любой такой подорграф̆ $G-w$ определяется следующим условием: $\lambda(G-w)=\max _{v \in V(G)} \lambda(G-v)$. Если орграф̆ $G-w$ не является сильно связныл, то большой интерес представляют его сильно связные подорграфы, числа Перрона которых совпадают с $\lambda(G-w)$. В спектралннй теории графов они называются сильными компонентами Перрона или, просто, компонентами Перрона. Все результаты нашей работы вытекают из следующей леммы.

ЛЕмма. Пусть $G$ - сильно связный орграф, $G-w$-любой максимальный подорграф с наибольшим числом Перрона, $(G-w)^{\prime}-$ любая его компонента Перрона и $G_{w}-$ подорграф, порожденныи всеми вершинами $G$, которые не принадлежат $(G-w)^{\prime}$. Тогда существуют две такие вершины $w_{\text {in }} u w_{\text {out }}$ в $G_{w}$, что все дуги из $V\left((G-w)^{\prime}\right)$ в $V\left(G_{w}\right)$ входят в вершину $w_{\text {in }}$ и все дуги из $V\left(G_{w}\right)$ в $V\left((G-w)^{\prime}\right)$ вьходят из вершинь $w_{\text {out. }}$. Вершина $w_{\text {out }}$ достижима из $w_{\text {in }}$ в $G_{w}$, и расстояние между $w_{\text {in }}$ и $w_{\text {out }}$ на единичy меньше порядка $G_{w}$.

Из леммы следует, что если орграф $G-w$ не является сильно связным, то степень исхода вершины $w_{\text {in }}$ равна единице. Поэтому если количество дуг, выходящих из каждой вершины сильно связного орграфа $G$, больше или равно двум, то любой его максимальный подорграф с наибольшим числом Перрона является сильно связным (этот результат уточняет лемму 6.5.2 [3]). В случае, когда $G$-сильно связный турнир с $n \geqslant 4$ вершинами, расстояние между $w_{\text {in }}$ и $w_{\text {out }}$ неможет быть болшше единицы. Если $w_{\text {in }}$ не совпадает с $w_{\text {оut }}$ (в противном случае $w_{\text {in }}=w_{\text {out }}=w$ ), то степень захода $w_{\text {in }}$ и степень исхода $w_{\text {out }}$ равны $n-2$. Так как существует дуга $\left(w_{\text {in }}, w_{\text {out }}\right)$, то любой максимальный подтурнир, отличньй от $G-w_{\text {in }}$ и $G-w_{\text {out }}$, является сильно связным (этот резултат уточняет теорему $3[4]$ ).

В силу леммы в случае произвольного сильно связного орграфа $G$ в его подорграффе $G w$ существует единственная гамильтонова иепь $\tilde{\gamma}=\left\{w_{i}\right\}_{i=0}^{p}$ такая, что $w_{0}=w_{\text {in }}$ и $w_{p}=w_{\text {out }}$. Если $\left(w_{m}, w_{k}\right) \in E(G)$, где $m \leqslant k-2$, то в $G-w_{m+1}$ путь $v_{0}, w_{0}, \ldots, w_{m}, w_{k}, \ldots, w_{p}, v_{p}$ соединяет вершины $v_{0}$ и $v_{p}$ сильно связного орграфа $(G-w)^{\prime}$ и проходит через вершины $w_{\text {in }}$ и $w_{\text {out }}$, которые не принадлежат $(G-w)^{\prime}$. Поэтому $\lambda\left(G-w_{m+1}\right)>\lambda(G-w)$, что противоречит выбору $w$ (на этом рассуждении основано доказательство леммы). Таким образом, помимо дуг $\left(w_{i}, w_{i+1}\right)$, где $i=0, \ldots, p-1$, подорграф $G_{w}$ может содержать только дуги вида $\left(w_{m}, w_{k}\right)$, где $0 \leqslant k<m \leqslant p$. Если $G$ - эйлеров орграф, то такие дуги отсутствуют и, следовательн, подорграф $G_{w}$ является направленной цепью.

Маршрут, проходящий через все вершины орграфа, назьвается гамильтоновым. Орграфф является сильно связным, если и только если он содержит циклический гамильтонов мариpym. Нетрудно показать, что в этом случае по крайней мере два максимальных подорграфа имеют гамильтоновы маршруты. При помощи гамильтоновой цепи $\tilde{\gamma}=\left\{w_{i}\right\}_{i=0}^{p}$ в $G_{w}$ можно построить гамильтонов маршрут в максимальном подорграфе $G-w$ с наибольшим числом Перрона (отсюда, в частности, будет следовать, что если $G$ - связный неориентированньй граф,, то $G-w$ также связен). Действительно, так как $w \in V\left(G_{w}\right)$, то $w=w_{m}$ для некоторого $m$, где $0 \leqslant m \leqslant p$. Выберем любые две такие вершины $v_{\text {in }}$ и $v_{\text {out в }}(G-w)^{\prime}$, что $\left(v_{\text {in }}, w_{\text {in }}\right) \in E(G)$ и

Работа выполнена при частичной поддержке Российского фонда фундаментальных исследований (гранты № 00-01-00153 и № 01-01-06046). 
$\left(w_{\text {out }}, v_{\text {out }}\right) \in E(G)$. Тогда объединение единственного пути из $w_{m+1}$ в $w_{\text {out }}$, любого гамиль-

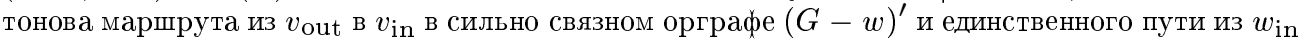
в $w_{m-1}$ является гамилштоновым маршрутом в $G-w$. Любой гамильтонов маршрут в $G-w_{\text {in }}$ заканчивается в $(G-w)^{\prime}$. Аналогично, любой гамильтонов маршрут в $G-w_{\text {out }}$ начинается в $(G-w)^{\prime}$. Хорошо известно ([5; гл. ХIII, теорема 7], см. также [6; следствие 3.5$\left.]\right)$, что матрица смежности орграфа с единственной компонентой Перрона имеет правый (левый) положительньй вектор Перрона (т.е. собственньй вектор с положительньми координатами, соответствующий числу Перрона), если и только если из любой его вершины можно добраться до компоненты Перрона (любая его вершина достижима из компоненты Перрона). Поэтому справедливо следующее утверждение.

ТЕОРема 1. Пусть $G$ - сильно связный орграф. Предположим, что каждый его максимальный подорграф $G-w$ с наибольшим числом Перрона содержит только одну компоненту Перрона. Тогда гамильтонова цепь из $w_{\text {in }} \theta$ out $\theta$ $G_{w}$ является единственным простым путем $\tilde{\gamma}=\left\{w_{i}\right\}_{i=0}^{p}$, проходящим через вершину $w$ и обладающим следующими свойствами:

1) для любого $i=0, \ldots, p$ максимальньй подорграф $G-w_{i}$ имеет наибольиее число Перрона;

2) матрица смежности $G-w_{0}$ имеет правый положительный вектор Перрона;

3) матрица смежнности $G-w_{p}$ имеет левый положительный вектор Перрона;

4) матрица смежности $G-w_{i}$, где $i=1, \ldots, p-1$, не имеет ни левого, ни правого положительного вектора Перрона.

Орграф с единственной компонентой Перрона является сильно связньм, если и только если его матрица смежности имеет правый и левьй положительные векторы Перрона. Поэтому в силу теоремы 1 максимальные подорграфы $G-w_{0}, \ldots, G-w_{p}$ все вместе наследуют свойства сильно связного орграфа.

ТЕОРема 2. Пусть $G$ - сильно связный орграф. Предположим, что некоторый максимальный подорграф $G-w$ с наибольшим числом Перрона обладает по крайней мере двумя компонентами Перрона. Тогда для любого $w_{\text {in }}$ существует единственная вершина $v$ такая, что $\left(v, w_{\text {in }}\right) \in E(G)$. Расстояние межуд $w_{\text {in }}$ и $v$ на единицу меньше порядка орграфа $G$.

Таким образом, при невыполнении условия теоремы 1 орграф̆ $G$ является гамильтоновьм и его диаметр на единицу меньше его порядка.

\section{СПИСОК ЛИТЕРАТУРЫ}

[1] А. М. Богомолов, В. Н. Салий. Алгебраические основы теории дискретных систем. M.: Наука, 1997. [2] С. В. Савченко // УМН. 2000. T. 55. № 1. С. 191-192. [3] R. A. Brualdi, B. Shader. Matrices of Sign-Solvable Linear Systems. Cambridge: Cambridge Univ. Press, 1995. [4] J. W. Moon. Topics on Tournaments. New York: Holt, Rinehart and Winston, 1968. [5] Ф. Р. Гантмахер. Теория матриц. М.: Наука, 1967. [6] H. Schneider // Linear Algebra Appl. 1986. V. 84. P. 161-189. 